\title{
Assessment of Factors Affecting Inactivated COVID-19 (CORONAVAC) Vaccine Response and Antibody Response in Healthcare Professionals
}

Hülya Özkan Özdemir ( $\sim$ drhulyaozkan@gmail.com )

University of Health Sciences, Izmir Bozyaka Education and Research Hospital

\section{Selma Tosun}

University of Health Sciences, Izmir Bozyaka Education and Research Hospital

Seher Ayten COŞKUNER

University of Health Sciences, Izmir Bozyaka Education and Research Hospital

Seval Demir

University of Health Sciences, Izmir Bozyaka Education and Research Hospital

\section{Research Article}

Keywords: COVID-19, CoronaVac, inactivated COVID-19 vaccine, antibody

Posted Date: May 20th, 2021

DOI: https://doi.org/10.21203/rs.3.rs-506854/v1

License: (c) (i) This work is licensed under a Creative Commons Attribution 4.0 International License.

Read Full License 


\section{Abstract}

\section{Background}

Although COVID-19 pandemic Phase III trial results of many vaccines were reported, the literature about community results is inadequate. This study aims to evaluate the experience gained during the vaccination process of healthcare workers $(\mathrm{HCW})$, the measured antibody responses and the factors affecting the response, and to contribute to the literature in this field by presenting the data.

Method

Anti-RBD anti-SARS Cov2 IgG antibodies were measured by the ELISA method in blood samples taken at least a month after the second vaccine from $264 \mathrm{HCW}$ vaccinated twice with an interval of 28 days. Information from individuals were collected with an online participation form.

Results

$264 \mathrm{HCW}$ (166 females (63\%), 98 males (37\%)) were included in the study whose age are between 23-69 (mean $44.22 \pm 11.58)$. After vaccination, $22(8.3 \%)$ were unresponsive, $25(9.5 \%)$ had weak response, and $217(82.2 \%)$ had a response. The overall rate of weak response and the high response was $91.6 \%$. In our study, that the antibody response was found to be statistically significantly lower in males ( $p: 0.022)$, there was a significant decrease in antibody response with advanced age $(p<0.005)$, and the difference was highly significant (p: 0.0005) above the age of 60.

\section{Conclusion}

In this study, 91.6\% anti spike antibodies were detected with CoronaVac which an inactivated vaccine and the antibody response is was lower in cases of advanced age, male gender, not having COVID-19, not developing PVAE, and having pandemic H1N1 vaccine.

\section{Background}

The clinical picture which attracted attention with the clustering of pneumonia cases in China in the last month of 2019 and whose causative agent was defined as a new coronavirus (SARS-CoV-2), leads to severe acute respiratory syndrome (table). It affected the whole world quickly and was declared a pandemic by the World Health Organization, named COVID-19, in February 2020. As of April 9, 2021, there are 134,621,148 confirmed cases and 2,917,645 deaths worldwide, and the pandemic still continues by making peaks all around the world (1-5).

While the personal protection measures, protective equipment usage, hand hygiene and social isolation rules that have been recommended to be practised from the beginning are beneficial, vaccination studies against the agent have also been started because they are not adequate to stop the pandemic. Vaccines having particular features have been developed by many companies. Preclinical and clinical studies on 
nearly 300 candidate vaccines are still ongoing worldwide. the vaccine of many companies in the world was started to be used with emergency use approval by the national authorities: FDA in the USA, EMA in Europe, China-India-England and Russia at the end of 2020. However, no COVID-19 vaccine had conventional approval for use yet. Therefore, efficacy and reliability studies regarding different vaccines are still ongoing $(6-10)$.

Coronaviruses are, structurally pleomorphic, enveloped viruses with characteristic S-protein spikes on their surface. The primary objective of all COVID-19 vaccines is to produce neutralizing antibodies against the $S$ protein of the virus in individuals. The $S$ protein of SARS CoV-2 is the most suitable antigen for the formation of neutralizing antibodies against this pathogen. It has two subunits consisting of $\mathrm{S} 1$ (NTD, RBD, and RBM domains) and S2 (FP, HR1 and 2 domains). S protein has a dynamic structure; It has two different structures as prefusion and post-fusion (11). Inactivated vaccines which are among the COVID-19 vaccines are chemically inactivated vaccines produced in Vero cell culture. Today, other inactivated vaccine studies are still continuing in China, India and Kazakhstan in addition to the CoronaVac produced by Sinovac in China and the BBIBP-CorV vaccines produced by Sinopharm in China. These vaccines are often combined with aluminum or a similar adjuvant aiming to stimulate the immune response. The target of inactivated vaccines includes not only the spike protein but all components of the virus (12-15). In our country, a Phase III study took place which started on September 14, 2020, regarding the inactivated vaccine named CoronaVac produced by Sinovac company in China. Following this, the vaccination of healthcare workers was started on January 12, 2021, after the emergency use approval. Subsequently, vaccination of individuals at risk in the community continues gradually.

Neutralizing antibodies formed after vaccination cannot be routinely examined due to the need for special laboratory infrastructure. The most scientific measurement of antibody response for coronaviruses other than neutralizing antibodies is recommended as the measurement of $\lg M$ and $\lg G$ against S1 protein $+\mathrm{N}$ protein (16). Although it is known that the detected spike antibodies (anti-S $\lg G$ ) correlate with the neutralizing antibody level, there is no standard threshold value for significant antibody titer; Therefore, there are difficulties in the interpretation of the results. Additionally, test kits produced by different companies result in different reference ranges. Moreover, since the methods of these test kits are different, it is not possible to compare the antibody results. However, these results are still suggestive, and it is expected that much more reliable results will be get as a result of standardization of test kits over time.

Antibody testing before vaccination in mass vaccination is not recommended as it is technically difficult, time-consuming and costly. Antibody screening after vaccination is not also recommended as a routine method but may be done for academic purposes. The main reasons for this are that the detection of neutralizing antibodies showing essential protection after vaccination requires special equipment, experience, time and cost and therefore it cannot be applied widely. In addition, measurement of antibodies in serum only shows the $B$ lymphocyte response, and it is not possible to measure and show the activity of $\mathrm{T}$ lymphocytes expressing memory cells with this method, but it is predicted that $\mathrm{T}$ 
lymphocytes are stimulated, and memory cells are being expressed/have expressed in COVID-19 vaccines just as in many vaccines (17-18).

This study aimed to measure the anti-spike antibody responses 28 days after the last dose of the inactivated COVID-19 vaccine (CoronaVac) which was administered to healthcare workers in our country at a dose of $3 \mu \mathrm{g}$ twice in 28 days and to evaluate the factors associated with antibody response.

\section{Methods}

In our country, the first vaccinations to healthcare workers were given on January 12, 2021, and the second dose of vaccines was completed between February 14-19. Approval for our study was obtained from the Ministry of Health Scientific Committee and İzmir Bozyaka Clinical Research Ethics Committee. Blood samples were acquired 28 days after the second vaccination of the individuals with their written consents and an online questionnaire was utilized to them at the same time. In the online form, age, gender, weight, height, chronic diseases and medicines, other vaccines they had, COVID-19 disease status, PVAE developing after COVID-19 vaccine were asked. Afterwards, anti-RBD antiSARS Cov2 IgG antibodies (Quantivac-Euroimmun/Germany) were measured in blood samples taken by the ELISA method. Obtained online survey data and measured antibody values were recorded.

The detected results are classified according to the recommendations of the test kit manufacturer as follows;

$\mathrm{BAU} / \mathrm{ml}$ : Binding antibody unit

$<25.6 \mathrm{BAU} / \mathrm{ml}$ Negative (no response)

25.6-35.1 BAU/ml limit value (weak response)

$>35.2 \mathrm{BAU} / \mathrm{ml}$ Positive (responsive)

\section{Statistical analysis:}

Statistical assessment was done using IBM SPSS, Version 22.0, software. Descriptive analyses were presented with mean and standard deviation for continuous variables, and numbers and percentages for categorical variables. Pearson's chi-square test was used in the evaluation of categorical variables, and $p$ $<0.05$ was accepted as the statistical significance level in all analyses.

\section{Results}

264 HCW (166 females (63\%), 98 males (37\%)) were included in the study whose ages are between 2369 (mean $44.2 \pm 11.58$ ). The mean age of men is 46.9 and women are 42.6 .73 of the participants had COVID-19 at any time before they were vaccinated. Profession distribution; There are 117 doctors, 79 
nurses, 47 laboratory workers and health officers, 21 office workers and security guards, and the education level of 231 (87.5\%) of those vaccinated is university and college.

After vaccination, 22 (8.3\%) were unresponsive, 25 (9.5\%) had weak response, and $217(82.2 \%)$ had response. The total number of people with weak and high responses was 242 with a rate of $91.6 \%$.

While a statistically significant difference was found between antibody response and gender, age, covidage, previous H1N1 pandemic vaccine and post-vaccination adverse effects (PVAE); No relationship was found between the presence of chronic diseases or an immunosuppressive condition, regular medication use, having another vaccine such as influenza, pneumococcus, tetanus, and hepatitis in the last year, and the body mass index (BMI) of the individuals (Table 1).

Table 1

Factors reducing or not affecting antibody response to COVID-19 vaccine

\begin{tabular}{|ll|}
\hline Factors/parameters reducing the COVID-19 vaccine antibody response & p-value \\
\hline Male gender & 0.007 \\
\hline Age increase & $<0.05$ \\
\hline 50 age & 0.021 \\
\hline No Covid history & 0.0005 \\
\hline Having H1N1 pandemic vaccine & 0.015 \\
\hline No PVAE & 0.044 \\
\hline Factors/parameters not affecting the COVID-19 vaccine antibody response & $\mathbf{p}-\mathbf{v a l u e}$ \\
\hline Presence of chronic diseases & 0.011 \\
\hline Presence of an immunosuppressive status & 0.138 \\
\hline Regular medication use & 0.398 \\
\hline Having influenza vaccination in the last year & 0.751 \\
\hline Having pneumococcal vaccine in the last year & 0.587 \\
\hline Having hepatitis or tetanus vaccine in the last six months & 0.535 \\
\hline BMl & 0.281 \\
\hline
\end{tabular}

In our study, it was found that the antibody response was statistically significantly lower in men ( $\mathrm{p}$ : $0.022)$. There was a significant decrease in antibody response with advancing age $(p<0.005)$. While the difference was less when the age groups were divided into $23-49$ years and $>50$ years ( $p$ : 0.056$)$, the difference was found to be highly significant when grouping as 23-59 years and >60 years (p: 0.0005) (Table 2). 
Table 2

Antibody responses by age groups (23-59 years and $>60$ years old)

\begin{tabular}{|llll|}
\hline Age & Negative (No response) & Limit (weak) & Decent response \\
\hline $23-59(\mathrm{n}: 234)$ & $14(\% 6)$ & $22(\% 9.4)$ & $198(\% 84.6)$ \\
\hline$>60(\mathrm{n}: 30)$ & $\mathbf{8}(\% 26.6)$ & $3(\% 10)$ & $19(\% 63.3)$ \\
\hline Total & $22(\% 8.3)$ & $25(\% 9.5)$ & $217(\% 82.2)$ \\
\hline
\end{tabular}

Antibody responses were found to be statistically significantly higher in healthcare workers who had COVID-19 infection at any time before vaccination (p: 0.017). Only one individual who had COVID-19 before was found to have a negative antibody and this individual is 61 years old and had COVID-19 disease in November 2020.

Five individuals, three males and two females, who were vaccinated after having the disease with weak antibody response were detected and their ages ranged between 35 to 48 . Additionally, it was determined that the antibody titres of the majority were high (35.2-99.9 BAU/ml in 23 people and $>100 \mathrm{BAU} / \mathrm{ml}$ in 44 people) when the results of people with COVID-19 vaccine and good response to antibody titers were examined in detail.

It was found that 68 of $73(93 \%)$ patients who had the disease were treated at home, four in hospitals and one in the intensive care unit. Therefore, no evaluation could be made about the relationship between antibody levels and clinical severity (mild-moderate-severe) of the disease.

No relationship was found between the presence of chronic disease and antibody response (p: 0.484) when the results of 103 people with chronic diseases were evaluated among the participants. Similarly, antibody unresponsiveness in patients who regularly use drugs due to chronic diseases is slightly higher than those who do not use drugs, but it was not statistically significant (p: 0.162). Once again, no significant difference was found in the antibody responses of 10 individuals who had immunosuppressive (malignancy or immunosuppressive drug use) for any reason than those who had not (p: 0.138).

No relationship was found between COVID-19 antibody responses with influenza vaccine (p: 0.919), pneumococcal vaccine (p: 0.044), and hepatitis or tetanus vaccine (p: 0.535) in the past year. However, it was determined that 54 of the people who participated in our study had a pandemic vaccine during the $\mathrm{H} 1 \mathrm{~N} 1$ pandemic in 2009. The antibody response developed with the COVID-19 vaccine was found to be lower in those individuals with the H1N1 vaccine, and the difference between those who were not in this group was found to be statistically significant (Spearman correlation coefficient: -0.139 , p-value: 0.024 ) (Table 3).

Since a weak negative correlation was detected between body mass index (BMI) and antibody levels, it was not statistically significant. 
Table 3

COVID-19 antibody responses in individuals with and without the H1N1 vaccine

\begin{tabular}{|llll|}
\hline H1N1 vaccination status (n:246)* & Negative & Limit & Positive \\
\hline Done (n:54) & $\mathbf{9 ( \% 1 7 )}$ & $5(\% 9)$ & $40(\% 74)$ \\
\hline Not done (n:192) & $\mathbf{1 2 ( \% 6 . 2 ) ~}$ & $18(\% 9.4)$ & $\mathbf{1 6 2}(\% 84.4)$ \\
\hline Total & 21 & 23 & 202 \\
\hline
\end{tabular}

*18 individuals do not remember if they got vaccinated

It was determined that antibody unresponsiveness was higher in people in whom PVAE was not observed, meaning that PVAE was observed slightly more in those with high antibody response ( $p$ : 0.011). When 73 healthcare workers who chose to be vaccinated despite having Covid were asked about their reasons for vaccination, it was learned that $51(70 \%)$ of them were not sure if protection would be provided by having the disease and thus, they were vaccinated to avoid having the disease again.

\section{Discussion}

The vaccine named CoronaVac which is applied in our country and evaluated the antibody responses after vaccination in our study is a vaccine containing aluminium hydroxide adjuvant produced by Sinovac company in China. The Phase I/II study was conducted in a single-centre, double-blinded, randomized placebo-controlled study in China with 743 individuals aged between 18-59, and the vaccine was found to be effective and safe as a result of a study (19). 144 individuals participated in the Phase I study of this vaccine, and $3-\mu \mathrm{g}$ and $6 \mu \mathrm{g}$ doses of vaccine were administered on two different schemes for 0-14 days and 0-28 days. In the study, antibody levels were between $46-50 \%$ in the blood samples obtained on the 14 th day, while it was $83 \%$ in the $3-\mu$ group and $79 \%$ in the $6-\mu \mathrm{g}$ group on the 28th day. Phase II study was conducted with 600 individuals in May 2020 and antibody responses were $92 \%$ for 3 $\mu \mathrm{g}, 98 \%$ for $6 \mu \mathrm{g}$ in the $0-14$-day scheme; it was found to $97 \%$ and $100 \%$ in the $0-28$ th day scheme, respectively.

In the Phase III study of the same vaccine conducted in Brazil, individuals both aged 18-59 and $>60$ years old were included (20). In the Phase III study in Brazil, $600 \mathrm{SU}(3 \mu \mathrm{g})$ vaccine was used and the 0-14day scheme was applied. 2270 (36.6\%) male, 3925 (63.4\%) female healthcare workers with a mean age of 39.42 participated in the study. The age distribution was recorded as 5879 individuals aged between 18-59 (94.9\%), 316 individuals aged between > 60 (5.10\%). The results of the Brazilian study were announced on January 7, 2021, and it was emphasized that the "clinical efficacy" level was reported as $78 \%$ and the "general efficacy" level as $50.4 \%$, while the rate of protection from mild illness was $78 \%$ and the rate of protection from severe illness was $100 \%$. About the low rate of effectiveness in this study, it has been explained as the high number of cases in the period when the study was conducted in Brazil and the detection of a large number of Covid patients in both the placebo and the study group. In 
addition, it was reported that the participants in the study were healthcare workers who were at a much higher risk for COVID-19, many parameters were accepted as positive, and even if they were not evaluated in other vaccine studies. For these reasons, it was estimated that its efficacy may have been relatively low (21-23).

CORONAVAC Phase III study conducted in Turkey has started on September 142020 and 24 centres participated. As of December 23 2020, 918 healthcare workers and 6453 individuals from the public (total 7371 people) were included, and the efficacy rate of the vaccine administered at a dose of $3 \mu \mathrm{g}$ with a 0 14-day scheme was reported as $91.25 \%$ (24).

There are other inactivated vaccine studies in China and different countries, and new studies are still ongoing $(25,26)$.

As it can be seen, while the efficacy of the vaccine was reported to be decent in Phase I-II studies regarding the vaccine named Coronavac which we evaluated in our study, different rates of responses were observed in other countries where phase III studies were conducted. In our study, when the response rate against the vaccine was evaluated, the weak response rate was $9.5 \%$ and the decent response rate was $82.2 \%$. One of the reasons for the different rates could be that the vaccine was administered with the 0-14 days scheme in some Phase III studies, and the 0-28 days scheme was used in this study. In addition, it was thought that this situation may have been observed due to different test kits and different measurement methods.

In our study, we found that the antibody response was weakened with advancing age, and this difference increased more significantly over the age of 60 . Although higher rates were reported in the Phase studies conducted by Wu et al. in individuals over 60 years old, this difference could be occurring due to the fact that our analysis was performed with the ELISA method and Wu's study was performed with the PRNT method, and our number of people over 60 years old was lower. However, we still think that more realistic data can be obtained, and the issue of age should be considered by following up the larger number of vaccinated individuals over the age of 60 . The concept of immune aging is rather complex and there is no validated method for measuring its degree. With advancing age, the numerical value of $T$ cells capable of responding to a vaccine and the survival rate of T cells, especially CD8 T cells, decreases significantly. B cells, on the other hand, although their number does not decrease much with age, less functional antibodies are produced due to the decreased expression of certain proteins. Therefore, lower antibody responses can be expected in the elderly $(27,28)$.

In our study, it was also found that being male was associated with low antibody response. As it is known, there is evidence that the immunological response to antigen may differ between genders. In women, the number and activity of innate immune cells such as monocytes, macrophages, and dendritic cells are higher than in men. Thus, responses to both infections and vaccines can be higher in women compared to men. Women have a higher proportion of CD4 + T cells and CD4 +/CD8 + T cells rate; $C D 8+$ $\mathrm{T}$ cell count is also lower than men. B cell count, IgG and IgM are similar in adulthood in both genders. 
Adult women typically have a high inflammatory cell-mediated immune response to vaccines which may explain the better effectiveness of vaccines in women than in men (29-31).

There was no emphasis on gender in any of the Phase studies conducted on CoronaVac, and we think that the higher vaccine response in women identified in our study should be considered when monitoring larger cohorts.

As an interesting data, it was determined that 54 of the healthcare professionals participating in our study had pandemic vaccine during the H1N1 pandemic in 2009, and antibody unresponsiveness developed against COVID-19 vaccine was found to be slightly higher in these individuals ( $p: 0.024)$. Although the number is fewer, we think that this issue should be evaluated in more detail in other studies. Again, in our study, it was determined that antibody unresponsiveness was higher in people who did not have PVAE, meaning that PVAE was slightly higher in those with high antibody response ( $p: 0.011)$. This situation may be elucidated by the lower rate of development or sense of PVAE in those with lower immune responses.

In our study, those who had COVID-19 and, then, the vaccine had higher antibody titres in accordance with our expectations. Certain studies demonstrated that the antibody response in people is reduced after the COVID-19 infection, especially in those who are asymptomatic. In a study about SARS, it was reported that the antibodies developed decreased over time, and this decrease accelerated after the 16th month and even disappeared in the third year $(32,33)$. It is difficult to predict how long the antibodies will continue to be presented in those who have the disease, and whether there will be a difference in the duration of antibody persistence in those vaccinated after the disease, and it is not clear for now. We anticipate that this issue will be clarified with long-term follow-up.

As with many infectious diseases that can be prevented by vaccination, it seems important to vaccinate a significant part of the community with available and accessible vaccines, as well as protective measures such as hand washing, mask-distance to protect from COVID-19 infection. Since inactivated vaccines are developed with conventional methods and do not carry live virus particles, they are considered safer. However, individuals vaccinated with inactivated vaccines should also be monitored in terms of Antibodydependent enhancement (ADE) and Vaccine-associated enhanced respiratory disease (VAERD) as in other COVID-19 vaccines $(34,35)$. Additionally, it is not yet clear whether the formation of strong antibodies against the inactivated vaccine will protect people from COVID-19 infection or how long this protection will continue. Moreover, it should be kept in mind that the antibody levels measured in serum against the vaccine only show the $B$ lymphocyte response, the $T$ cell activity representing the memory cells is also very important in the response to the vaccine, and the sensitivity of these tests will be lower than the neutralization test results with PRNT.

This study had some limitations, primarily that we did not have the information about the previous vaccination antibody levels. The previous antibody levels would have sitrengthen our results. The sample size is limited therefore the size increase may affect mean results. 
Strength of the study is that all our sample consists of the health professionals therefore all COVID-19 PCR records and COVID-19 medical records were well known. Thus, the finding of our study is stronger.

\section{Conclusion}

During the COVID-19 pandemic which still poses a risk for the whole world, it is still uncertain if the vaccines obtained emergency use approval are protective and how long their protection will continue, and the answers to these questions can be given after the conclusion of the ongoing Phase studies and monitoring the real-life data. However, the data obtained indicate that protection occurs regardless of which vaccine is used. Although the effectiveness of vaccines produced by various production techniques is different, it is estimated that the adverse effects will be less because all of the virus (all components) is used by inactivating inactivated vaccines, and it also includes variant strains even if the antibody response is lower. In our study, it was determined that $91.6 \%$ anti spike antibodies were detected with CoronaVac which is an inactivated vaccine, and the antibody response was lower in cases with advanced age, being male, not having COVID-19, not developing PVAE, and having been given pandemic $\mathrm{H} 1 \mathrm{~N} 1$ vaccine. Our study is also planned to be conducted six months and 12 months after vaccination, and we think that long-term antibody responses will be more enlightening.

\section{Declarations}

\section{Acknowledgements}

We are thankful to Dr. Murat AYSIN and Dr. Alpay ARI for their support in the statistical analysis and interpretation of the data.

Authors' contributions HÖÖ conceived the study, undertook the data collection, and constructed the paper, ST conceived the study, assisted with the statistical analysis and interpretation of the results. SAC and SD collected the dat. All authors read and approved the manuscript.

\section{Institutions at which the work was performed:}

University of Health Sciences, Bozyaka Education and Research Hospital, Department of Clinical Microbiology and Infectious Diseases, İzmir-Turkey

Disclosure (Authors): The authors declare no conflicts of interest.

\section{Disclaimer:None}

\section{Funding}

Not applicable

\section{Conflict of interest}


The authors declare that they have no conflict of interest.

\section{Ethical approval and consent to participate}

The study was approved by Bozyaka Education and Research Hospital Ethical Committee of the University of Health Sciences (2021/27.10.02.2021). All methods were performed in accordance with the relevant guidelines and regulations. Written informed consent was obtained from all of the participants during the survey

\section{Consent for publication}

Not applicable

\section{Availability of data and material}

The dataset used and analysed during the current study are available from the corresponding author on reasonable request.

\section{Code availability}

Not applicable

\section{References}

1. World Health Organization. Director-General's remarks at the media briefing on 2019-nCoV on February 11 2020. http://www.who.int/dg/speeches/detail/who-director-general-s-remarks-at-themedia-briefing-on-2019-ncov-on-11-february-2020 (Accessed on March 14, 2021).

2. WHO corona virüs disease (COVID-19) dashboard. 2020. https://covid19.who.int/ (accessed March 14, 2021).

3. Li J, Huang DQ, Zou B, Yang H, Hui WZ, Rui F, Yee NTS, Liu C, Nerurkar SN, Kai JCY, Teng MLP, Li X, Zeng H, Borghi JA, Henry L, Cheung R, Nguyen MH. Epidemiology of COVID-19: A systematic review and meta-analysis of clinical characteristics, risk factors, and outcomes. J MedVirol. 2021 Mar;93(3):1449-1458. doi: 10.1002/jmv.26424. Epub 2020 August 25. PMID: 32790106; PMCID: PMC7436673.

4. World HealthOrganization (2020) Novelcoronavirus (2019-nCoV). Situation Report 22. https://www.who.int/emergencies/diseases/novel-coronavirus-2019/situation-reports (accessedMarch 14, 2021).

5. https://www.worldometers.info/coronavirus/

6. US FoodandDrug Administration. Development and licensure of VaccinestoPrevent COVID-19: Guidanceforlndustry. https://www.fda.gov/media/139638/download (Accessed on March 14, 2021).

7. WHO. Draftlandscape of COVID-19 candidate vaccines. https://www.who.int/publications/m/item/draft-landscape-of-covid-19-candidate- 
vaccines(accessed March 14, 2021).

8. https://clinicaltrials.gov/ct2/results? recrs=\&cond=covid+vaccine\&term=\&cntry=\&state=\&city=\&dist=

9. Kathryn M Edwards, KM, Orenstein WA. Coronavirusdisease 2019 (COVID-19): Vaccinestoprevent SARS-CoV-2 infection. update (Access March 14, 2021)

10. Kim JH, Marks F, Clemens JD. Looking beyond COVID-19 vaccine phase 3 trials, Nature Medicine, February 2021; Vol 27; 205-211. https://doi.org/10.1038/s41591-021-01230-y

11. Kaur SP, Gupta V. COVID-19 Vaccine: A comprehensivestatusreport. VirusRes. 2020 Oct15;288:198114. doi: 10.1016/j.virusres.2020.198114. Epub 2020 Aug 13. PMID: 32800805; PMCID: PMC7423510.

12. Gao Q, Bao L, Mao H, Wang L, Xu K, Yang M, Li Y, Zhu L, Wang N, Lv Z, Gao H, Ge X, Kan B, Hu Y, Liu J, Cai F, Jiang D, Yin Y, Qin C, Li J, Gong X, Lou X, Shi W, Wu D, Zhang H, Zhu L, Deng W, Li Y, Lu J, Li C, Wang X, Yin W, Zhang Y, Qin C. Development of an inactivated vaccine candidate for SARS-CoV-2. Science. 2020 July 3;369(6499):77-81. doi: 10.1126/science.abc1932. Epub 2020 May 6. PMID: 32376603; PMCID: PMC7202686.

13. Krammer F. SARS-CoV-2 vaccines in development. Nature. 2020 Oct;586(7830):516-527. doi: 10.1038/s41586-020-2798-3. Epub 2020 September 23. PMID: 32967006.

14. Iversen PL, Bavari S. Inactivated COVID-19 vaccinestomake a global impact. LancetInfectDis. 2021 Feb3:S1473-3099(21)00020-7. doi: 10.1016/S1473-3099(21)00020-7. Epubahead of print. PMID: 33548196; PMCID: PMC7906657.

15. Muruato AE, Fontes-Garfias CR, Ren P, Garcia-Blanco MA, Menachery VD, Xie X, Shi PY. A highthroughput neutralizing antibody assay for COVID-19 diagnosis and vaccine evaluation. NatCommun. 2020 August 13;11(1):4059. doi: 10.1038/s41467-020-17892-0. PMID: 32792628; PMCID: PMC7426916.

16. Lee P, Kim CU, Seo SH, Kim DJ. current status of COVID-19 Vaccine Development: Focusing on Antigen Design and clinical trials on later stages. ImmuneNetw. 2021 Feb 26;21(1):e4. doi: 10.4110/in.2021.21.e4. PMID: 33728097; PMCID: PMC7937514.

17. Kim JH, Marks F, Clemens JD. Looking beyond COVID-19 vaccine phase 3 trials. NatMed. 2021 Feb;27(2):205-211. doi: 10.1038/s41591-021-01230-y. Epub 2021 January 19. PMID: 33469205.

18. Soleimanpour S, Yaghoubi A. COVID-19 vaccine: where are we now and where should we go? ExpertRevVaccines. 2021 Jan;20(1):23-44. doi: 10.1080/14760584.2021.1875824. Epub 2021 February 17. PMID: 33435774; PMCID: PMC7898300.

19. Zhang Y, Zeng G, Pan H, Li C, Hu Y, Chu K, Han W, et al. Safety, tolerability, and immunogenicity of an inactivated SARS-CoV-2 vaccine in healthy adults aged 18-59 years: a randomised, double-blind, placebo-controlled, phase 1/2 clinical trial. LancetInfectDis. 2021 Feb;21(2):181-192. doi: 10.1016/S1473-3099(20)30843-4. Epub 2020 November 17. PMID: 33217362; PMCID: PMC7832443.

20. Palacios R, PatiñoEG, de OliveiraPiorelli R, Conde MTRP, Batista AP, Zeng G, Xin Q, et al. Double-Blind, Randomized, Placebo-ControlledPhase III Clinical Trial toEvaluatetheEfficacyandSafety of treating 
Healthcare ProfessionalswiththeAdsorbed COVID-19 (Inactivated) VaccineManufacturedbySinovac PROFISCOV: A structured summary of a study protocol for a randomised-controlled trial. Trials. 2020 October 15;21(1):853. doi: 10.1186/s13063-020-04775-4. PMID: 33059771; PMCID: PMC7558252.

21. Vilches TG, Rubio FA, Perroni RAF, Almeida GB, Fortaleza CMCB, Ferreira CP. Vaccination efforts in Brazil: scenarios and perspectives under a mathematical modeling approach. MedRxivhttps://doi.org/10.1101/2021.02.22.21252208;February 2021.

22. N. Scalzaretto, "Coronavac has overallefficacy of 50.4 percent, saysbutantan," Jan 2021. [Online]. Available: https://brazilian.report/coronavirus-brazil-live-blog/ 2021/01/12/coronavac-has-overallefficacy-of-50-4-percent-says-butantan/

23. https://brazilian.report/coronavirus-brazil-live-blog/2021/01/12/coronavac-has-overall-efficacy-of50-4-percent-says-butantan/ BBC. Sinovac: BrazilresultsshowChinesevaccine 50.4\% efective. https:// www.bbc.com/news/world-latin-america-55642648 (2020).

24. https://sol.org.tr/haber/sinovac-covid-19-asisinin-faz-iii-sonuclarini-acikladi25600 https://www.aa.com.tr/tr/koronavirus/coronavac-asisinin-guvenilirlik-ve-etkililigi-turkiyeninfaz-3-on-raporuyla-ortaya-kondu/2089235 http://www.sinovac.com/?optionid=754\&auto_id=922

25. Xia S, Duan K, Zhang Y, Zhao D, Zhang H, Xie Z, Li X, et al. Effect of an inactivated vaccine against SARS-CoV-2 on safety and immunogenicity outcomes: Interim analysis of 2 randomized clinical trials. JAMA. 2020 Sep 8;324(10):951-960. doi: 10.1001/jama.2020.15543. PMID: 32789505; PMCID: PMC7426884.

26. Xia S, Zhang Y, Wang Y, Wang H, Yang Y, Gao GF, Tan W, et al. Safetyandimmunogenicity of an inactivated SARS-CoV-2 vaccine, BBIBP-CorV: a randomised, double-blind, placebo-controlled, phase 1/2 trial. LancetInfectDis. 2021 Jan;21(1):39-51. doi: 10.1016/S1473-3099(20)30831-8. Epub 2020 October 15. PMID: 33069281 ; PMCID: PMC7561304.

27. Cunha LL, Perazzio SF, Azzi J, Cravedi P, Riella LV. Remodeling of the immune response with aging: immune senescence and its potential impact on COVID-19 immune response. Front Immunol 2020; 11: 1748 .

28. Jeyanathan M, Afkhami S, Smaill F, Miller MS, Lichty BD, Xing Z. Immunologicalconsiderationsfor COVID-19 vaccine strategies. NatRevImmunol 2020; 20: 615-32.

29. Ghosh S, Klein RS. Sexdrivesdimorphicimmuneresponsestoviralinfections. J Immunol. 2017 March 1;198(5):1782-1790. doi: 10.4049/jimmunol.1601166. PMID: 28223406; PMCID: PMC5325721.

30. Klein SL, Flanagan KL. Sex differences in immune responses. NatRevlmmunol. 2016 Oct;16(10):62638. doi: 10.1038/nri.2016.90. Epub 2016 August 22. PMID: 27546235.

31. Flanagan KL, Fink AL, Plebanski M, Klein SL. SexandGenderDifferences in the outcomes of Vaccinationoverthe Life Course. AnnuRev Cell Dev Biol. 2017 Oct6;33:577-599. doi:

10.1146/annurev-cellbio-100616-060718. PMID: 28992436.

32. Long QX, Tang XJ, Shi QL, et al. Clinicalandimmunologicalassessment of asymptomatic SARS-CoV2 infections. NatMed. Published online June 18, 2020. doi:10.1038/s41591-020-0965-6 
33. Cao WC, Liu W, Zhang PH, Zhang F, Richardus JH. The disappearance of antibodies to SARSassociated coronavirus after recovery. N Engl J Med. 2007;357(11):1162-1163. doi:10.1056/ NEJMc070348

34. Eroshenko N, Gill T, Keaveney MK, Church GM, Trevejo JM, Rajaniemi H. Implications of antibodydependent enhancement of infection for SARS-CoV-2 countermeasures. NatBiotechnol. 2020;38(7):789-791. doi:10.1038/s41587-020-0577-1

35. Graham BS. Rapid COVID-19 vaccinedevelopment. Science. 2020;368(6494):945-946. doi:10.1126/science.abb8923 